\title{
A Radio Frequency Magnetoelectric Antenna Prototyping Platform for Neural Activity Monitoring Devices with Sensing and Energy Harvesting Capabilities
}

\author{
Diptashree Das ${ }^{1}$, Mehdi Nasrollahpour ${ }^{1}$, Ziyue Xu ${ }^{1}$, Mohsen Zaeimbashi ${ }^{2}$, \\ Isabel Martos-Repath ${ }^{1}$, Ankit Mittal ${ }^{1}{ }^{\mathbb{D}}$, Adam Khalifa ${ }^{3}$, Sydney S. Cash ${ }^{3}(\mathbb{D}$, \\ Aatmesh Shrivastava ${ }^{1}$, Nian X. Sun ${ }^{1}$ and Marvin Onabajo ${ }^{1, *(D)}$ \\ 1 Department of Electrical and Computer Engineering, Northeastern University, Boston, MA 02115, USA; \\ das.d@northeastern.edu (D.D.); nasrollahpourmotla.m@northeastern.edu (M.N.); \\ xu.ziy@northeastern.edu (Z.X.); martos-repath.i@northeastern.edu (I.M.-R.); \\ mittal.ank@northeastern.edu (A.M.); aatmesh@ece.neu.edu (A.S.); n.sun@northeastern.edu (N.X.S.) \\ 2 The Wellman Center for Photomedicine, Massachusetts General Hospital, Harvard Medical School, \\ Boston, MA 02114, USA; mzaeimbashi@mgh.harvard.edu \\ 3 Department of Neurology, Massachusetts General Hospital, Harvard Medical School, \\ Boston, MA 02114, USA; akhalifa1@mgh.harvard.edu (A.K.); scash@mgh.harvard.edu (S.S.C.) \\ * Correspondence: monabajo@ece.neu.edu; Tel.: +1-617-373-3452
}

Received: 10 November 2020; Accepted: 8 December 2020; Published: 11 December 2020

\begin{abstract}
This article describes the development of a radio frequency (RF) platform for electromagnetically modulated signals that makes use of a software-defined radio (SDR) to receive information from a novel magnetoelectric (ME) antenna capable of sensing low-frequency magnetic fields with ultra-low magnitudes. The platform is employed as part of research and development to utilize miniaturized ME antennas and integrated circuits for neural recording with wireless implantable devices. To prototype the reception of electromagnetically modulated signals from a sensor, a versatile Universal Software Radio Peripheral (USRP) and the GNU Radio toolkit are utilized to enable real-time signal processing under varying operating conditions. Furthermore, it is demonstrated how a radio frequency signal transmitted from the SDR can be captured by the ME antenna for wireless energy harvesting.
\end{abstract}

Keywords: magnetoelectric sensor; magnetoelectric antenna; software-defined radio (SDR); electromagnetic modulation; energy harvesting

\section{Introduction}

Wireless devices with minuscule size, low power requirements, and low cost are in high demand for a variety of operating frequencies and emerging applications. Magnetoelectric (ME) antennas enable these features and are feasible for compact biomedical and wearable devices with the capability to sense small magnetic field variations at low amplitude (picoTesla to milliTesla) and low frequencies (DC to several kilohertz), which is typically a challenging task due to the abundance of environmental noises in this frequency range.

Figure 1 visualizes some of these magnetic field sensing applications, which include vector fluxgate magnetometers on aircrafts such as unmanned aerial vehicles and microsatellites [1], Internet-based home energy tracking [2], wireless vehicle detection and traffic surveillance [3], and wireless brain/body implantable devices [4]. As a step towards the practical integration of ME antennas into embedded 
systems, this article introduces the incorporation of a software-defined radio (SDR) for the measurement of low-frequency fields with ME sensors having resonance frequencies from tenths to hundredths of megahertz [5] and for efficient power transfer at gigahertz frequencies using ultra-compact $\mathrm{ME}$ antennas [6]. Since these ME sensors consist of nanoelectromechanical (NEMS) ME antennas, they are attractive for integrated devices that monitor neural activity through magnetic field variations with low frequency and low amplitude [4]. Similar to magnetic inductors with high quality factor [7], ME antennas can be designed with dimensions as small as 100s of micrometers and high quality factor (>100) [8]. ME antennas can exhibit three possible resonance modes across the length, width, and thickness depending on their fabrication, where the width and thickness resonance modes are predominantly utilized [6]. As a consequence of their unique design, the devices can be realized with dual resonant modes having two operating frequencies: a thin-film bulk acoustic wave resonator (FBAR) high-frequency $(2.5 \mathrm{GHz})$ resonance mode associated with thickness, and a nano-plate resonator (NPR) low-frequency $(<215 \mathrm{MHz})$ resonance mode associated with width [9]. The dimensions of the ME antenna used for the experiments here were $250 \mu \mathrm{m} \times 174 \mu \mathrm{m}$ with measured quality factors of 161 for the low-frequency sensing operation and 113.2 for the high-frequency energy harvesting operation. Another ME antenna from a different fabrication run was characterized in [6], where the reported values for the return loss, gain, directivity, and efficiency of the $\mathrm{ME}$ antenna were $10.26 \mathrm{~dB}$, $-18 \mathrm{dBi}, 6 \mathrm{~dB}$, and $0.403 \%$, respectively. Thus far, the small size of the ME antenna has made it challenging to design an antenna array with a high-frequency mode of operation. However, for the low-frequency NPR mode, it is feasible to fabricate an antenna array on one wafer such that each antenna has a different resonant frequency [6].

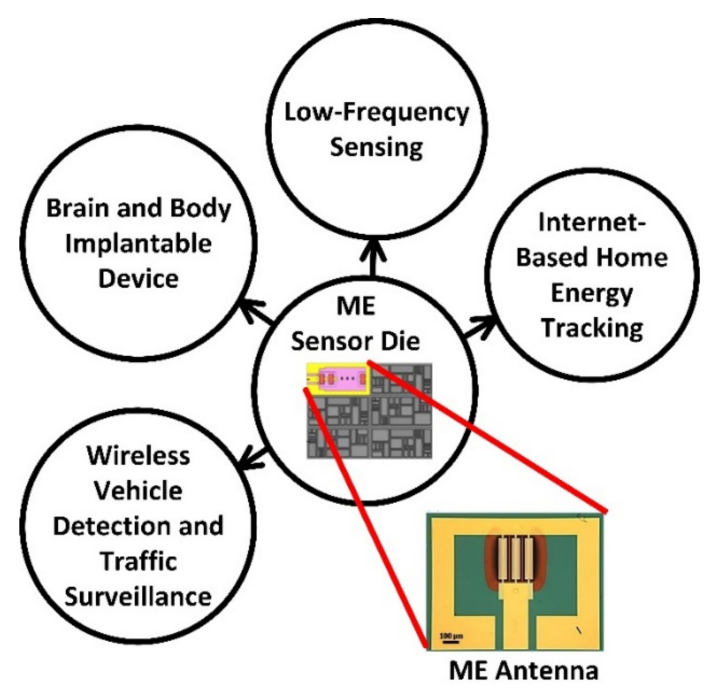

Figure 1. Potential magnetoelectric (ME) sensor applications.

The first steps towards integrating ME antennas with complementary metal-oxide semiconductor (CMOS) circuits were reported in [10]. In this paper, we discuss a platform that is part of the design process to evaluate and establish wireless links with ME antennas for the development of nanoscale neural radio frequency identification (NanoNeuroRFID) devices for brain implantable devices (IMDs) [4]. The NanoNeuroRFID chip in Figure 2 contains three major components for magnetic neural signal sensing, modulation, and transmission: a circuit to drive the ME antenna, an energy harvesting circuit for power management, and a timing control circuit. The SDR is utilized as the external transceiver that provides wireless power transfer at a relatively high radio frequency (RF) to the NanoNeuroRFID, which will perform functions for wireless power transmission at an intermediate RF frequency under the impact of neural modulation at the ME antenna. As shown in Section 3, the SDR can also receive the modulated signal to extract the information from the magnetic sensing of the low-frequency neural signals. The human brain consists of several major layers $[11,12]$. 
The external transceiver is placed outside the scalp, and the NanoNeuroRFID with its integrated ME antenna is used as an epicortical implant as depicted in Figure 2. An analysis of the path loss between the external transceiver and the envisioned implantable device can be found in [4]. Similar to the power amplifier testing platform in [13], a hardware-software co-development platform was created for the prototyping and optimization of the communication and energy transfer links with ME antennas. The portable Universal Software Radio Peripheral (USRP) B200mini-i SDR (Ettus, Santa Clara, CA, USA) supports a wide range of frequencies $(60 \mathrm{MHz}$ to $6 \mathrm{GHz})$, which makes it suitable for this platform with regards to the frequencies of interest for resonant-based sensing and wireless energy harvesting using ME antennas, as depicted in Figure 2. Note that, even though the antenna exhibits magnetic field sensing and energy harvesting capabilities at both resonant modes, the low-frequency NPR mode has shown better sensing performance during experiments, whereas the high-frequency FBAR mode has shown better wireless power transfer efficiency. The resonance frequency of the NPR mode is a function of the resonator width, Young's modulus $(\Delta \mathrm{E})$, and density of the piezoelectric thin film, whereas the resonance frequency of the FBAR mode depends on the thickness of the resonator, $\Delta \mathrm{E}$, and the density of the piezoelectric thin film [6]. The thickness of the piezoelectric film is much smaller than its width, and $\Delta \mathrm{E}$ contributes different levels of strain on the piezoelectric material across the thickness and width of the rectangular ME antenna. At high frequency, $\Delta \mathrm{E}$ causes more strain on the piezoelectric material, causing better suitability for wireless energy harvesting and outperforming the width mode [9]. Conversely, the magnetic material strain for sensing is mostly contributed along the width of the ME antenna. Hence, the low-frequency NPR mode exhibits better sensitivity for the sensing operation. For the sensing of low-frequency neural signals, we created a circuit simulation model [14] that accounts for the amplitude modulation of signals emitted by ME antennas when excited by AC magnetic fields. In this article, we describe for the first time how this sensed ME field information can be received and demodulated with an SDR. The GNU Radio interface enables the SDR to reconstruct the signal that causes low-frequency modulation of the carrier signal. For energy harvesting, on the other hand, the SDR acts as an RF source to transfer power to the ME antenna. This paper illustrates both applications with specific test conditions and measurements. Section 2 provides information on the SDR-based development platform and test setups. Section 3 includes measurement results, based on which conclusions are made in Section 4.

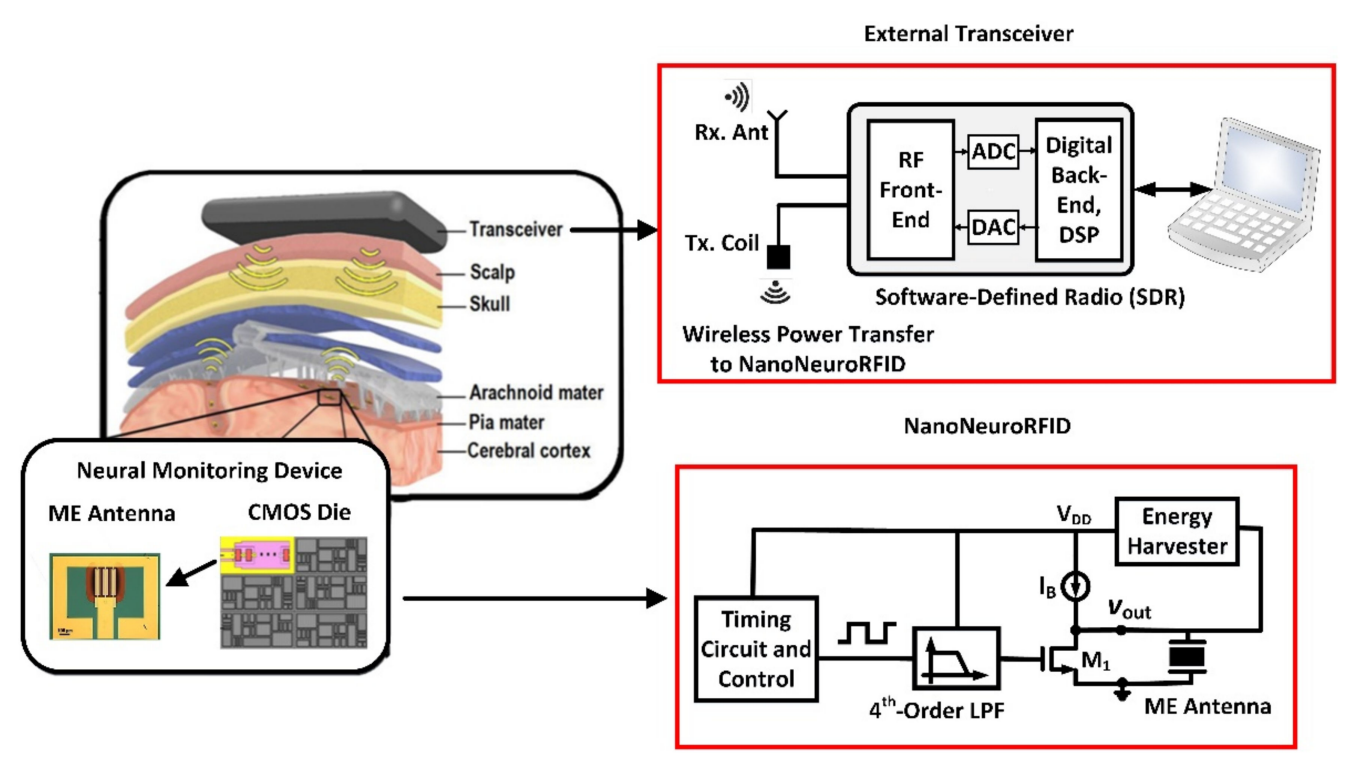

Figure 2. Illustration of magnetic field sensing with an ME antenna, nanoscale neural radio frequency identification (NanoNeuroRFID), and a software-defined radio (SDR). Acronyms: analog-to-digital converter (ADC), digital-to-analog converter (DAC), digital signal processor (DSP), and low-pass filter (LPF). 


\section{Test Platform}

\subsection{SDR-Based Approach}

A primary use of the test platform shown in Figure 3 is to obtain further experimental confirmation for the reception of electromagnetically modulated signals with an ME antenna as established through the simulation model in [14]. The field-programmable gate array (FPGA) based SDR provides the flexibility to evaluate receiver capabilities and digital filtering requirements prior to the design of smaller customized integrated circuits. In general, an ME-antenna-based sensor produces a magnetically modulated signal under the influence of an AC magnetic field and a DC biasing field, such that the AC field with a frequency of $f_{m}$ creates components in the spectrum around the carrier frequency $f_{c}$, which can be observed at $f_{c} \pm f_{m}$ [14]. An ME antenna can be fabricated for different frequency ranges according to the demand of a specific application. The SDR-based receiver allows the user to control the center frequency, receiver gain, channel bandwidth, and sampling frequency simultaneously in the software domain via the Universal Software Radio Peripheral (USRP). Hence, it enables a flexible RF platform for reliable reception of the incoming signal. The received signal is further processed by the digital signal processor (DSP) of the SDR, which allows adaptation via programming within the GNU Radio framework. For the experimental evaluation of the receiver operation, the SDR was tuned to $135 \mathrm{MHz}$ in alignment with the low-frequency resonance mode of the ME sensor under investigation. On the other hand, the SDR was tuned to $2.515 \mathrm{GHz}$ for the high-frequency resonance mode energy harvesting operation.

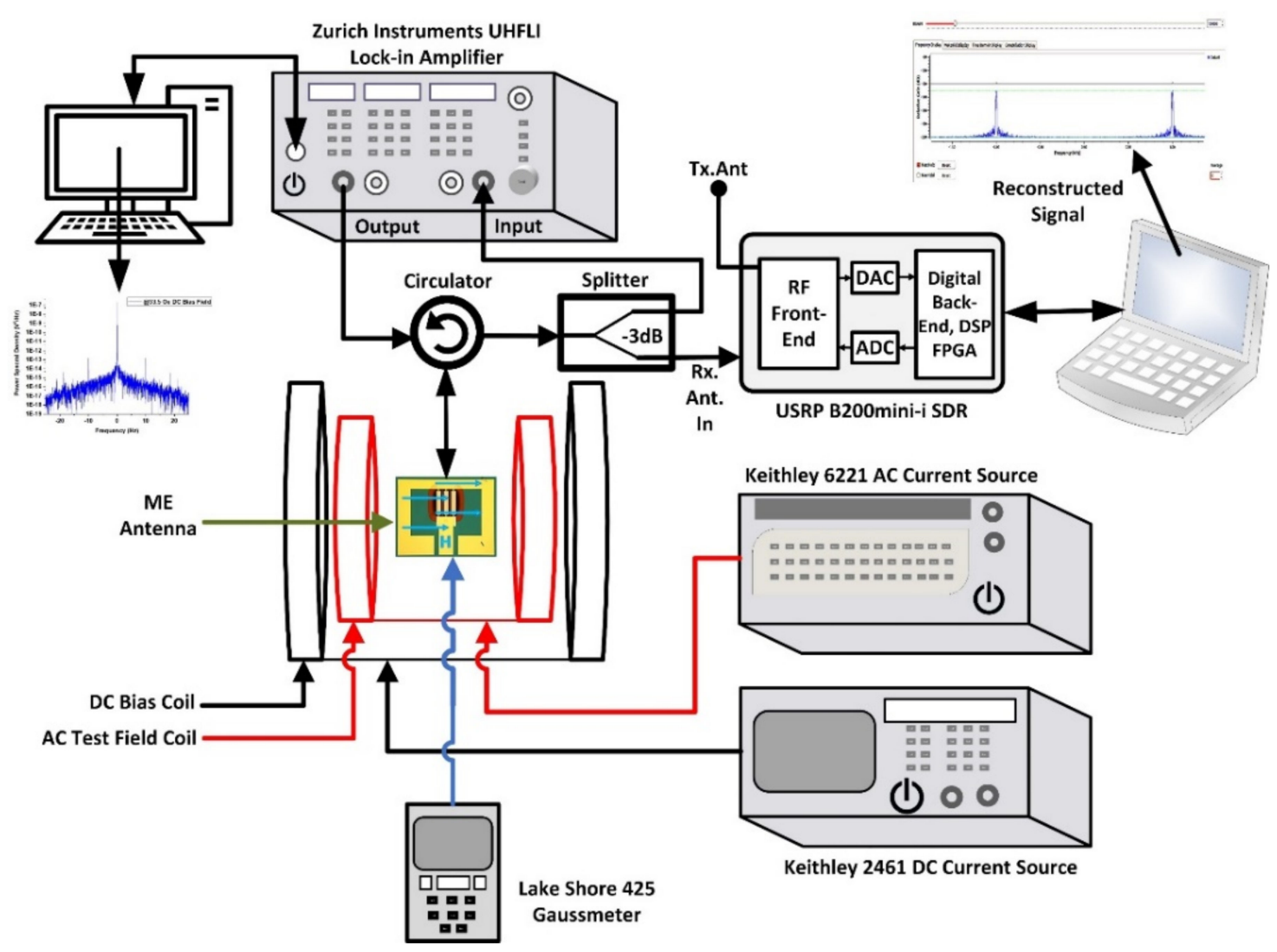

Figure 3. Test setup for the SDR receiver operation with the ME antenna as a magnetic field sensor.

\subsection{Receiver Prototyping}

Figure 3 displays the experimental setup for low-frequency magnetic field sensing using an ME antenna as a sensor and an SDR as a receiver. The magnetic sensing was initiated via a strain response 
of the magnetic material in the ME antenna when exposed to an external AC magnetic field. An AC current source with a controllable magnitude and frequency generated the AC field through a small Helmholtz coil, whereas a DC bias field was applied through a DC current source connected to a larger Helmholtz coil. The orientation of the DC bias field was placed perpendicular to the length of the resonator to maximize the magnetoelectric coupling coefficient and sensitivity. The alternating magnetic field was in the same direction as the DC bias field. Furthermore, the ME antenna was excited by a sinusoidal voltage from the ultra-high-frequency lock-in (UHFLI) amplifier at a certain carrier frequency. Due to the ME effect and the non-linear properties of the magnetic material, the ME sensor produced an amplitude modulated signal [14]. The circulator and splitter configuration in Figure 3 were used to receive the returned signal from the ME sensor with the SDR, while simultaneously monitoring it with the lock-in amplifier. SubMiniature version A (SMA) connectors and cables were utilized for the connections in the measurement setup. The magnetically amplitude-modulated received signal was amplified and down-converted by the RF front-end of the SDR and further processed in its DSP to extract the signal components that carry the ultra-low-magnitude AC magnetic field information sensed by the ME antenna.

\subsection{Energy Harvesting Experiment}

The experimental setup for the verification of the energy harvesting characteristics is portrayed in Figure 4, where the transmission (Tx) coil and ME antenna were organized with the help of two 3D-printed plastic manipulators to accurately adjust their positions in the $x, y$, and $z$ directions with a spatial resolution of less than $200 \mu \mathrm{m}$. The use of plastic manipulators (instead of metallic ones) reduces the effects of interference and its impact on Tx coil impedance matching. To transfer power, the SDR produces a pulse-modulated signal with a variable amplitude and duty cycle that excites the Tx coil at the same frequency $(2.515 \mathrm{GHz})$ as the high-frequency resonance mode of the ME antenna. The single-turn Tx coil on a printed circuit board (PCB) on Flame Retardant 4 (FR4) substrate was optimized and fabricated with a capacitive L-matching network to transfer maximum power at $2.515 \mathrm{GHz}$ [9]. SMP connectors have lower parasitic capacitance compared to SMA connectors. Due to this advantage, an SMP connector was soldered onto the Tx coil PCB to connect it to a vector network analyzer (VNA) in order to confirm impedance matching at $2.515 \mathrm{GHz}$ prior to the experiment. During the testing, the power was wirelessly transferred from the Tx coil to the ME antenna. First, the power transfer efficiency (PTE) of the ME antenna was measured by making use of the experimental setup depicted in Figure 4a. The PTE measurement did not involve the energy harvesting chip designed as part of this research. This measurement was performed to assess the performance of the ME antenna by directly connecting it to a spectrum analyzer (SA) with an SMA cable. Next, the setup in Figure $4 \mathrm{~b}$ was utilized to investigate and optimize system-level energy harvesting with the Tx coil, ME antenna, and a custom-designed energy harvesting chip. Here, the SDR acted as a variable RF source, allowing for the adjustment of the transmission in GNU Radio to control four main parameters of the pulse-modulated signal to produce the expected transient response during the energy harvesting test: the transmission gain of the SDR, amplitude of the signal, time period of the pulse-modulated signal, and pulse width. The energy harvesting chip shown in Figure $4 \mathrm{~b}$ contains a basic single-ended modified Dickson AC-to-DC rectifier with off-chip passive components for impedance matching. It was fabricated in $130 \mathrm{~nm}$ CMOS technology, and the die was directly wire-bonded to the PCB. An oscilloscope was used to measure the transient response (voltage vs. time) of the energy harvesting operation. 


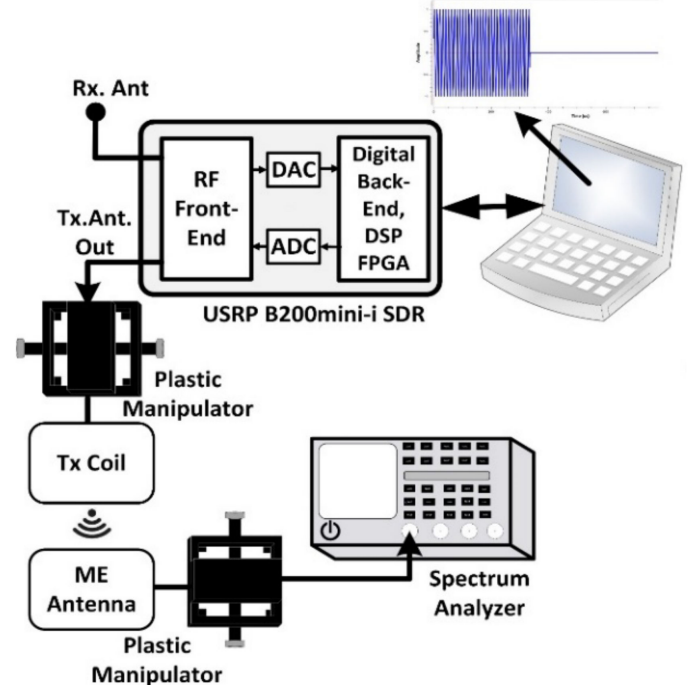

(a)

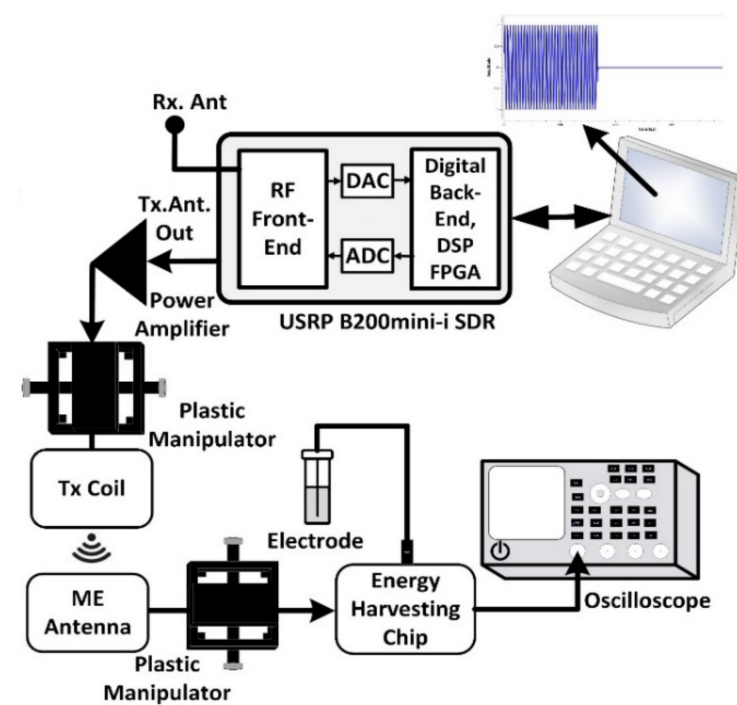

(b)

Figure 4. Experimental setup for energy harvesting with an ME antenna excited by the SDR: (a) test arrangement for power transfer efficiency (PTE) measurements; (b) test arrangement to characterize the energy harvesting capability.

\section{Experimental Results}

\subsection{SDR-Based Receiver Testing}

Figure 5 visualizes the signal processing flow of the SDR receiver within the USRP and GNU Radio, which implemented the receiving operation for the sensing with ME antennas described in Section 2.2. Note that the same SDR was used as the transmitter for energy harvesting. Its output power is controlled by two parameters - the digital-to-analog converter setting (scalar value between 0 and 1) and the SDR gain setting [15]. To confirm the power level of the pulse-modulated carrier signal, the SDR output power was characterized first, particularly as a preparation for the energy harvesting test discussed in Section 2.3. Figure 6a includes the results from the SDR output power analysis using a spectrum analyzer (SA), which were obtained by varying the transmitter gain of the SDR for a particular frequency. Furthermore, the SDR was employed to generate an amplitude-modulated signal with a modulation component at a $1 \mathrm{kHz}$ offset from the $135 \mathrm{MHz}$ carrier frequency, which is the frequency that matches the ME antenna center frequency of the resonance mode used for sensing. This signal from the SDR transmitter was measured directly with a spectrum analyzer and with the SDR receiver path to assess the receiver operations and accuracy under weak amplitude modulation (69 $\mathrm{dB}$ below the carrier). The results in Figure $6 \mathrm{~b}$ reveal a $0.72-2.40 \mathrm{~dB}$ receiver error range with a dependence on the variable SDR transmitter gain and receiver gain (here $61 \mathrm{~dB}$ ). With transmitter gain settings above $70 \mathrm{~dB}$, the SDR receiver error relative to the spectrum analyzer measurement remained below $1.5 \mathrm{~dB}$.

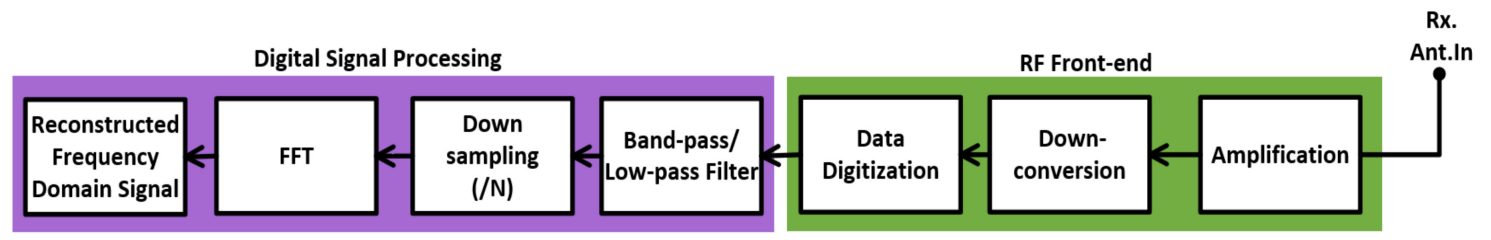

Figure 5. Overview of SDR hardware and software receiver operations. 


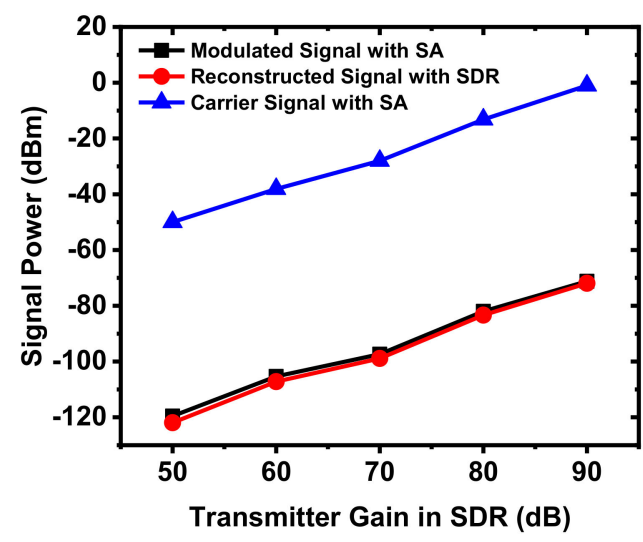

(a)

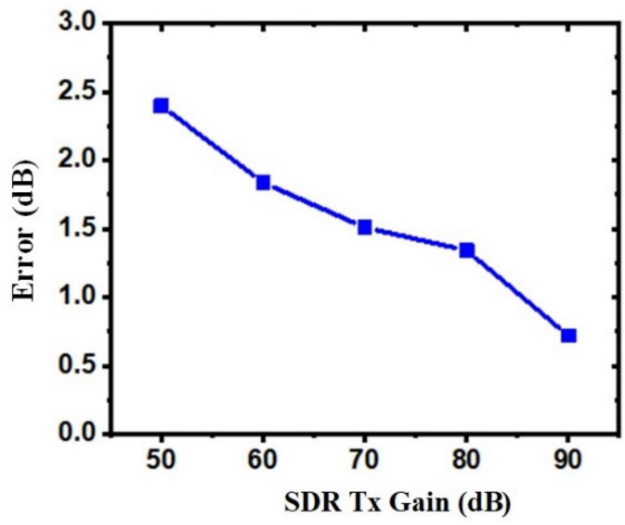

(b)

Figure 6. (a) Results from power measurements with a spectrum analyzer (SA) and the SDR (set to $61 \mathrm{~dB}$ receiver gain); (b) measured error vs. SDR Tx gain.

To prototype the sensing with the ME antenna under wired conditions as shown in Figure 3, the SDR parameters were configured for an RF center frequency of $135 \mathrm{MHz}$, a receiver gain of 16-49 dB, and a sampling rate of $1 \mathrm{MHz}$. As depicted in Figure 5, the received signal was amplified and down-converted to produce a 12-bit digitized baseband signal with in-phase and quadrature-phase components. The signal was then processed in the DSP unit to extract the amplitude modulation component with a band-pass filter centered around $1 \mathrm{kHz}$ with a $100 \mathrm{~Hz}$ bandwidth. Afterwards, the signal was down-sampled to reduce the sample rate by a factor of 5 prior to reconstructing it with a fast Fourier transform (FFT) having a length of 32,768.

Figure 7 displays the reconstructed frequency domain $1 \mathrm{kHz}$ signal component from the electromagnetic modulation of the ME antenna using the sensing test setup in Figure 3 . The reconstructed $1 \mathrm{kHz}$ frequency domain signal had a power of $-109.8 \mathrm{dBm}$ captured with a $61 \mathrm{~dB}$ SDR receiver gain. From the data in Figure 6a, the reconstructed $1 \mathrm{kHz}$ signal component, measured while using the SDR as a transmitter and receiver, had a power of $-107.3 \mathrm{dBm}$ with $61 \mathrm{~dB}$ SDR receiver gain. The $2.5 \mathrm{~dB}$ difference between these two scenarios is strongly influenced by the variations of losses in the passive RF components in Figure 3. Note that the attenuation due to losses can be compensated by increasing the SDR's receiver gain by approximately $2.5 \mathrm{~dB}$, which would require a sensor calibration measurement with a known magnetic field strength prior to using it. Table 1 summarizes the ME antenna and SDR receiver parameters and measurement results from the test set-up shown in Figure 3. Figure 8 displays the $1 \mathrm{kHz}$ time domain signal component captured after the SDR receiver operations. The $1 \mathrm{kHz}$ signal had a $2.1 \mu \mathrm{V}$ peak-to-peak voltage and was received with the same SDR receiver settings as described for the reconstructed frequency domain information.

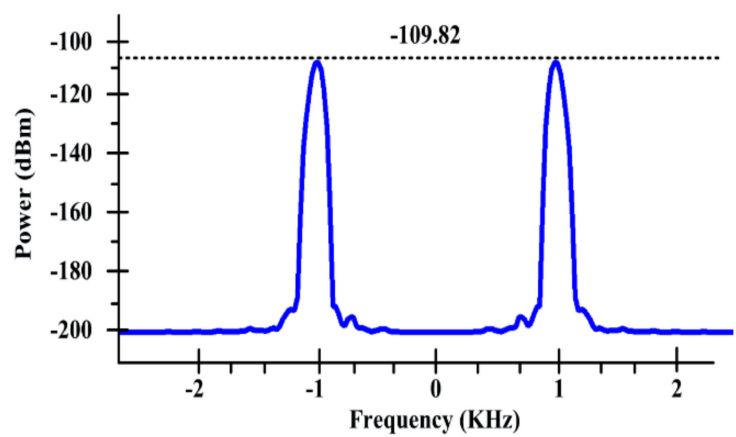

Figure 7. Reconstructed frequency domain signal captured from the $135 \mathrm{MHz}$ ME sensor by the SDR with $61 \mathrm{~dB}$ receiver gain. 
Table 1. SDR receiver parameters and measurement results.

\begin{tabular}{cc}
\hline Parameter & Value \\
\hline ME sensor frequency & $135 \mathrm{MHz}$ \\
ME antenna dimensions & $250 \mu \mathrm{m} \times 174 \mu \mathrm{m}$ \\
Modulation type & $\mathrm{AM}$ \\
Modulated signal frequency & $1 \mathrm{kHz}$ \\
Tx-Rx separation & $1 \mathrm{~m}$ \\
SDR Rx gain & $49-70 \mathrm{~dB}$ \\
SDR baseband sampling rate & $1 \mathrm{MHz}$ \\
CPU utilization rate & $35 \%$ \\
Carrier signal power at the spectrum analyzer & $-43.5 \mathrm{dBm}$ \\
Modulated signal power at the spectrum analyzer & $-103.3 \mathrm{dBm}$ \\
Reconstructed signal power at the SDR with 61 dB Rx gain & $-109.8 \mathrm{dBm}$ \\
\hline
\end{tabular}

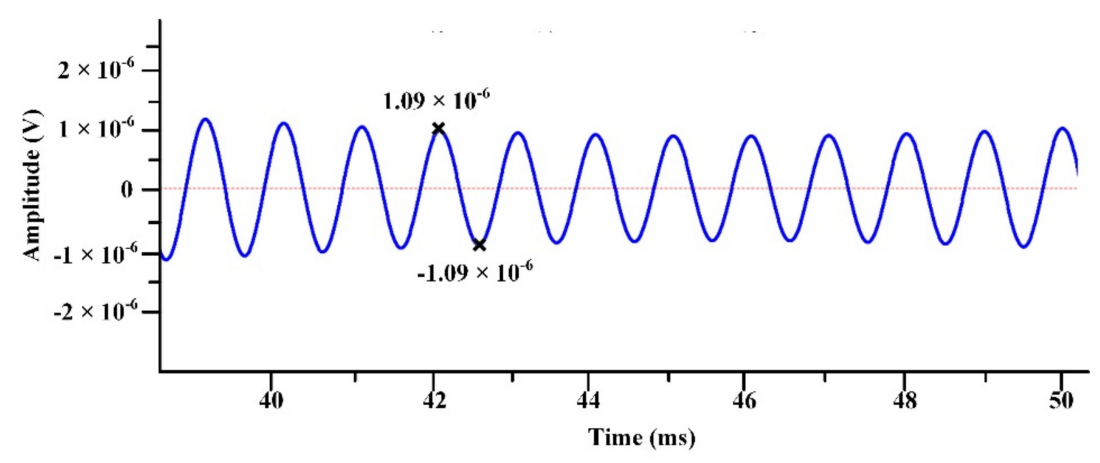

Figure 8. Time domain signal captured from the $135 \mathrm{MHz}$ ME sensor by the SDR with $61 \mathrm{~dB}$ receiver gain.

\subsection{SDR-Based Energy Harvesting Demonstration}

Here, the Tx coil was driven with the SDR providing a pulse-modulated carrier signal at the high-resonance mode frequency of the ME antenna using the setup in Figure 4 (with a $2.515 \mathrm{GHz}$ sinusoidal carrier). The pulse-modulated carrier signal was specified through GNU Radio such that it provided adequate ranges of the pulse time period, pulse width, duty cycle, carrier amplitude, and output power with a $50 \Omega$ termination: $1 \mathrm{~s}-100 \mathrm{~s}, 1 \mu \mathrm{s}-100 \mathrm{~s}, 0.005-100 \%, 100 \mathrm{mV}-1 \mathrm{~V}$, and $-60 \mathrm{dBm}-14 \mathrm{dBm}$, respectively.

\subsubsection{Power Transfer Efficiency of the ME Antenna}

The energy harvesting capability of the ME antenna can be investigated in terms of the power transfer efficiency (PTE) with respect to the distance between the Tx coil and ME antenna. The PTE is measured by taking the ratio of the power received by the ME antenna to the transmitted power from the Tx coil [8]. Figure 9a shows the PTE versus the distance between the Tx coil and ME antenna measured with the configuration in Figure 4a without the energy harvesting chip. The results demonstrate the capability to transmit power from the Tx coil driven by the SDR to the ME antenna connected to a spectrum analyzer. The impedance matching of the Tx coil was confirmed with a VNA measurement before performing the wireless transfer using the test setup. Figure 10 displays the $S_{11}$ versus frequency plot to confirm the impedance matching of the Tx coil at $2.515 \mathrm{GHz}$. The stimulated Tx coil at $2.515 \mathrm{GHz}$ generated an RF field with a magnetic flux density (Bx) ranging from 23 nanoTesla to 331 nanoTesla at the ME antenna, depending on the distance to the Tx coil. The described setup allows for characterizations of wireless power transfer in air. Note that the medium of the wireless transfer has an effect on the PTE. From the measurements with tissue in [9], it was observed that the PTE was actually 3.1 times higher than in air. More insights about this phenomenon, which is related to the higher relative permittivity of the tissue for the high-frequency FBAR mode $(2.51 \mathrm{GHz})$, can be found in [9]. Furthermore, the electromagnetic (EM) wave travels slower in the tissue medium. As a 
result, the effective wavelength becomes shorter and the EM wave can propagate even in the region very close to the Tx coil. In the current work, the RF magnetic field induced a strain in the ME antenna that triggered it to operate in the high-frequency mode. It is important to note that the PTE decreased with increasing distance between the Tx coil and ME antenna, as expected. The distance measurement range was selected as 3.5-13.5 mm, which is of interest for brain implantable devices based on the assessments in [4,9]. The measured PTE from this experiment was approximately $0.28 \%$ at $3 \mathrm{~mm}$ distance between the Tx coil and ME antenna in free space, which is one of the highest among the reported PTEs for receivers using antennas of small size [16-19]. The specific absorption rate (SAR) was not simulated because it is highly contingent on the application. The SAR has to be determined by characterizing the energy absorption over time. With relatively high applied power levels due to the PTE, the transmitted power can be duty cycled in such a way that the resulting SAR is within the limit imposed by the Federal Communications Commission (FCC). As a part of related research efforts, M. Zaeimbashi et al. reported in [9] that the PTE with an ME antenna allows a biomedical implantable device to be placed within the body in accordance with the FCC SAR regulation.

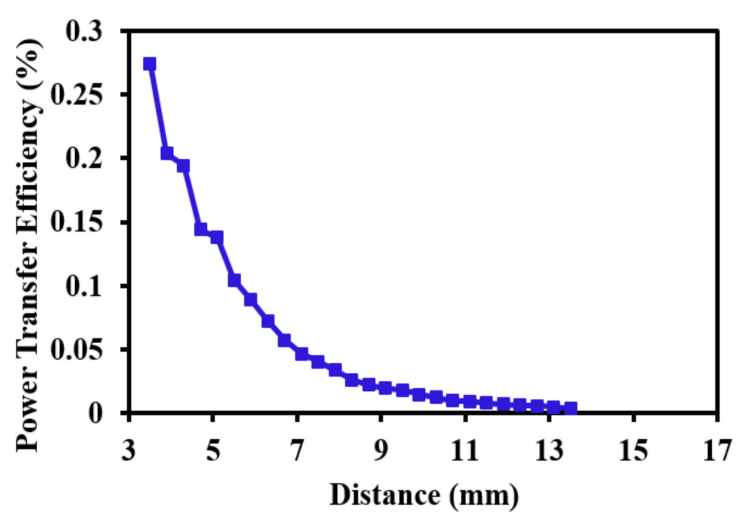

(a)

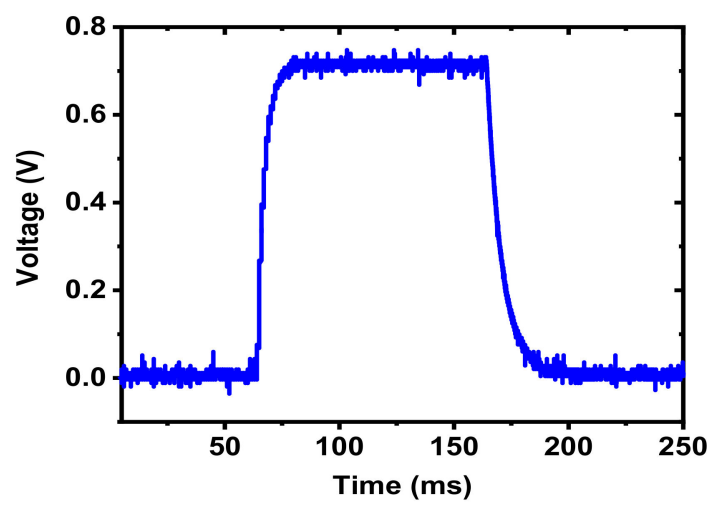

(b)

Figure 9. Energy harvesting measurements: (a) PTE vs. distance; (b) transient output with electrode load (100 ms Tx pulse width).

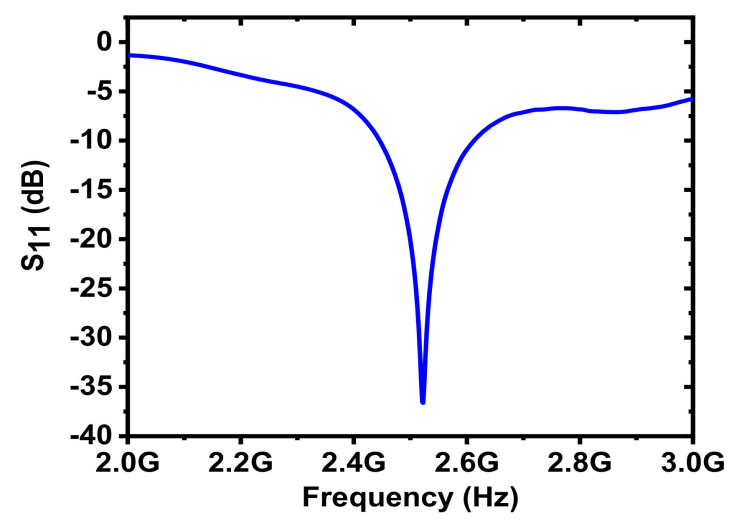

Figure 10. Measured $S_{11}$ of the Tx coil matched at $2.515 \mathrm{GHz}$.

\subsubsection{Measurements with the Energy Harvesting Chip}

Figure $9 \mathrm{~b}$ shows the transient response measured with the energy harvesting configuration in Figure $4 \mathrm{~b}$. Here, the pulse-modulated $2.515 \mathrm{GHz}$ carrier signal with a $100 \mathrm{~ms}$ pulse width and $1 \mathrm{~s}$ period led to $-1.1 \mathrm{dBm}$ signal power at the input of the energy harvester chip, producing an output voltage of $715 \mathrm{mV}$. During the testing, a predominantly capacitive electrode (PI20030.01A3 Pt/Ir, purchased from Microprobes, Gaithersburg, MD, USA) was connected as load, with a capacitance of approximately $4.5 \mathrm{nF}$. With the $45 \mathrm{~dB}$ power amplifier gain, the power delivered to the Tx coil 
was $17 \mathrm{dBm}$. The reduction to $-1.1 \mathrm{dBm}$ at the ME antenna output was mainly caused by path loss and finite PTE between the Tx coil and ME antenna. Other losses in the system came from the cables and plastic manipulator connectors. The transient response changed with different pulse widths and load conditions. With a $10 \mathrm{~ms}$ pulse width and $1 \mathrm{~s}$ time period, for example, the energy harvester provided a $505 \mathrm{mV}$ output under the same conditions. The transient response of the system also strongly depended on the resistor-capacitor constant of the rectifier on the energy harvesting chip and its load. Hence, the flexibility of the SDR allows us to investigate and optimize transmission parameters based on the characteristics of other system components.

\section{Conclusions}

The SDR-based hardware-software platform introduced herein can be utilized to prototype a receiver for the sensing of ultra-low magnetic fields with a magnetoelectric antenna. Furthermore, the same platform enables the evaluation of wireless power transfer to the antenna for the optimization of energy harvesting. The combination of an ME antenna with an integrated CMOS chip (together referred to as NanoNeuroRFID) is under development to perform neural recording based on magnetic sensing with small brain implants. The SDR described in this paper can serve as an external transmitter for wireless power transfer to the NanoNeuroRFID and as a receiver of the sensed information. The SDR-based approach provides experimental flexibility for practical assessments prior to the design of custom receivers and transmitter circuitry for IMDs on a PCB or chip.

Author Contributions: Conceptualization, D.D., M.N. and M.Z.; methodology, D.D., M.N., Z.X., M.Z. and A.K.; software, D.D.; validation, D.D., M.N. and Z.X.; formal analysis, D.D., M.N., Z.X. and M.O.; resources, M.N., Z.X., M.Z., I.M.-R., A.M. and A.K.; data curation, D.D.; writing一original draft preparation, D.D., M.O.; writing-review and editing, D.D., M.N., Z.X., M.Z., I.M.-R., A.M., A.K., S.S.C., A.S., N.X.S., M.O.; supervision, M.O., N.X.S., A.S., S.S.C. All authors have read and agreed to the published version of the manuscript.

Funding: This research was funded by National Institutes of Health (NIH) under grant \#1UF1NS107694-01.

Acknowledgments: The authors thank Gaurav Jha (Analog Devices, Inc.) and Francesco Restuccia (Northeastern University) for valuable discussions and suggestions.

Conflicts of Interest: The authors declare no conflict of interest.

\section{References}

1. Wang, J.; Chen, X. A fluxgate magnetometer for navigation and sensing: Noise character and digital filtering. In Proceeding of the IEEE Sensors, Busan, Korea, 1-4 November 2015.

2. Liu, X.; Lam, K.H.; Zhu, K.; Zheng, C.; Li, X.; Du, Y.; Liu, C.; Pong, P.W.T. Overview of Spintronic Sensors With Internet of Things for Smart Living. IEEE Trans. Magn. 2019, 55, 1-22. [CrossRef]

3. He, Z.; Zhu, H.; Yu, F. A vehicle detection algorithm based on wireless magnetic sensor networks. In Proceeding of the 4th IEEE International Conference on Information Science and Technology, Shenzhen, China, 26-28 April 2014.

4. Zaeimbashi, M.; Lin, H.; Dong, C.; Liang, X.; Nasrollahpour, M.; Chen, H.; Sun, N.; Matyushov, A.; He, Y.; Wang, X.; et al. NanoNeuroRFID: A Wireless Implantable Device Based on Magnetoelectric Antennas. IEEE J. Electromagn. RF Microw. Med. Biol. 2019, 3, 206-215. [CrossRef]

5. Nan, T.; Hui, Y.; Rinaldi, M.; Wang, X. Self-Biased 215MHz Magnetoelectric NEMS Resonator for Ultra-Sensitive DC Magnetic Field Detection. Sci. Rep. 2013, 3, 1985. [CrossRef] [PubMed]

6. Nan, T.; Lin, H.; Gao, Y.; Matyushov, A.; Yu, G.; Chen, H.; Sun, N.; Wei, S.; Wang, Z.; Li, M.; et al. Acoustically actuated ultra-compact NEMS magnetoelectric antennas. Nat. Commun. 2017, 8, 296. [CrossRef] [PubMed]

7. Gao, Y.; Zardareh, S.Z.; Yang, X.; Nan, T.X.; Zhou, Z.Y.; Onabajo, M.; Liu, M.; Aronow, A.; Mahalingam, K.; Howe, B.M.; et al. Significantly Enhanced Inductance and Quality Factor of GHz Integrated Magnetic Solenoid Inductors With FeGaB/A12O3 Multilayer Films. IEEE Trans. Electron Devices 2014, 61, 1470-1476.

8. Feld, D.; Parker, R.; Ruby, R.; Bradley, P.; Dong, S. After 60 years: A new formula for computing quality factor is warranted. In Proceeding of the 2008 IEEE Ultrasonics Symposium, Beijing, China, 2-5 November 2008. 
9. Zaeimbashi, M.; Nasrollahpour, M.; Khalifa, A.; Romano, A.; Liang, X.; Chen, H.; Sun, N.; Matyushov, A.; Lin, H.; Dong, C.; et al. Ultra-compact Dual-band Smart NEMS Magnetoelectric Antennas for Simultaneous Wireless Energy Harvesting and Magnetic Field Sensing. bioRxiv 2020, in press.

10. Nasrollahpour, M.; Romano, A.; Zaeimbashi, M.; Liang, X.; Chen, H.; Sun, N.; Emam, S.; Onabajo, M.; Sun, N. Integration of a novel CMOS-compatible magnetoelectric antenna with a low-noise amplifier and a tunable input matching. Analog. Integr. Circuits Signal Process. 2020, 105, 407-415. [CrossRef]

11. Wenger, C.; Salvador, R.; Basser, P.J.; Miranda, P.C. The electric field distribution in the brain during TTFields therapy and its dependence on tissue dielectric properties and anatomy: A computational study. Phys. Med. Biol. 2015, 60, 7339-7357. [CrossRef] [PubMed]

12. Lok, E.; San, P.; Hua, V.; Phung, M.; Wong, E.T. Analysis of physical characteristics of Tumor Treating Fields for human glioblastoma. Cancer Med. 2017, 6, 1286-1300. [CrossRef] [PubMed]

13. Chauhan, H.; Onabajo, M.; Kvartenko, V.; Coxe, R.; Weber, T. An Optimization Platform for Digital Predistortion of Power Amplifiers. IEEE Des. Test 2015, 33, 49-58. [CrossRef]

14. Martos-Repath, I.; Mittal, A.; Zaeimbashi, M.; Das, D.; Sun, N.X.; Shrivastava, A.; Onabajo, M. Modeling of magnetoelectric antennas for circuit simulations in magnetic sensing applications. In Proceedings of the 63rd International Midwest Symposium on Circuits and Systems (MWSCAS 2020), Springfield, MA, USA, 9-12 August 2020.

15. Zitouni, R.; George, L. Output power analysis of a software defined radio. In Proceeding of the IEEE Radio and Antenna Days of the Indian Ocean (RADIO 2016), St. Gilles-les-Bains, Reunion, 10-13 October 2016.

16. Khalifa, A.; Liu, Y.; Karimi, Y.; Wang, Q.; Eisape, A.; Stanacevic, M.; Thakor, N.; Bao, Z.; Etienne-Cummings, R. The Microbead: A $0.009 \mathrm{~mm} 3$ Implantable Wireless Neural Stimulator. IEEE Trans. Biomed. Circuits Syst. 2019, 13, 971-985. [CrossRef] [PubMed]

17. Ahn, D.; Ghovanloo, M. Optimal Design of Wireless Power Transmission Links for Millimeter-Sized Biomedical Implants. IEEE Trans. Biomed. Circuits Syst. 2016, 10, 125-137. [CrossRef] [PubMed]

18. Gao, L.; Yang, Y.; Brandon, A.; Postma, J.; Gong, S. Radio 488 frequency wireless power transfer to chip-scale apparatuses. In Proceedings of the MTT-S International 489 Microwave Symposium (IMS 2016), San Francisco, CA, USA, 22-27 May 2016.

19. Kuo, N.-C.; Zhao, B.; Niknejad, A. Equation-Based Optimization for Inductive Power Transfer to a Miniature CMOS Rectenna. IEEE Trans. Microw. Theory Tech. 2018, 66, 2393-2408. [CrossRef]

Publisher's Note: MDPI stays neutral with regard to jurisdictional claims in published maps and institutional affiliations.

(C) 2020 by the authors. Licensee MDPI, Basel, Switzerland. This article is an open access article distributed under the terms and conditions of the Creative Commons Attribution (CC BY) license (http://creativecommons.org/licenses/by/4.0/). 